\title{
Valsartan Attenuates Cardiotoxicity in Breast Cancer Patients After Chemotherapy
}

\author{
Nawal A Al-Hamadi ${ }^{1}$, Imad Al-Sabri' ${ }^{2,5}$, Amina B Aldujele ${ }^{3}$, Salam J Al-Fatlawi ${ }^{4}$ and Zuhair Allebban ${ }^{5 *}$ \\ ${ }^{1}$ Technical Institute of Kufa, Najaf, Iraq \\ ${ }^{2}$ Middle Euphrates Cancer Therapy Center, Najaf, Iraq \\ ${ }^{3}$ Department of Physiology, College of Medicine, Kufa University, Najaf, Iraq \\ ${ }^{4}$ Department of Community Medicine, College of Medicine/Kufa University, Najaf, Iraq \\ ${ }^{5 *}$ Middle Euphrates Unit for Cancer Research, College of Medicine/University of Kufa, Najaf, Iraq
}

\section{Abstract}

Objective: To determine whether valsartan would be useful in attenuating Adriamycin/Cyclophosphamide/ Herceptin ( $\mathrm{ACH}$ )-induced cardiotoxicity and to assess the role of biomarkers as indicators of cardiotoxicity in patients with breast cancer undergoing cancer therapy.

Background: Valsartan is indicated to reduce the risk of cardiovascular death and hospitalization in patients with chronic heart disease. The administration of cancer drugs, in particular ( $\mathrm{ACH})$ is associated with increased risk of cardiotoxicity. The use of herceptin improves survival of women with early-stage positive human epidermal growth factor receptor 2 (HER2) breast cancer but also adds substantially to cost, toxicity, and inconvenience. Little is known about the potential role of valsartan in the prevention of $\mathrm{ACH}$-mediated cardiotoxicity and the predictive role of biomarkers.

Method: A total of 60 untreated HER-2 positive breast cancer patients (ages $24-70 \mathrm{yr}$, mean $56 \mathrm{yr}$ ) were enrolled in this study and were divided to two groups. Group 1 and 2 had 30 patients each, and group 1 received $\mathrm{ACH}$ plus valsartan, group 2 received $\mathrm{ACH}$ alone. HER2- positive patients were recruited at the Cancer Therapy Center in Najaf, Iraq. Ejection fraction \% (EF\%), cardiac Troponin I (cTnl) and high sensitivity C-reactive protein (hsCRP) were measured at baseline and at six months after $\mathrm{ACH}$ administration.

Results: Chemotherapy induced significant transient increase in $\mathrm{EF} \%, \mathrm{cTnl}$, and hsCRP compared to baseline values $(P<0.001)$. Within one week after valsartan treatment, all these increases returned back to nearly normal levels. Notably, valsartan administration caused a significant decrease in cTnl and hsCRP values compared to baseline $(P<0.001)$ and it improved EF\% value.

Conclusion: This study demonstrated that prophylactic administration of valsartan resulted in the partial attenuation of cardiotoxicity caused by chemotherapy which was characterized by the improvement of EF\% and in lowering serum level of cTnl and hsCRP.

Keywords: Valsartan; Cardiotoxicity; Breast cancer; Cardio protection

\section{Introduction}

$\mathrm{ACH}$ administration has been shown to cause significant cardiotoxicity [1], which could show up immediately or weeks later. The cardiotoxicity can manifest itself as pericarditis-myocarditis syndrome, transient arrhythmias, and in severe cases, as cardiac decompensation $[2,3]$. Adriamycin is an anthracycline antibiotic with antineoplastic activity and a trade name of doxorubicin. It interacts with DNA by intercalation and used to treat various types of cancers including breast cancer. Cyclophosphamide is a cytotoxic alkylating agent, used as antineoplastic drug for a wide variety of cancers including breast cancer, it also used as an immunosuppressant to prevent graft rejection. Herceptin is a brand name of Trastuzumab, it is a monoclonal antibody used to treat breast cancer, specifically, HER2 receptor positive. ACH may be used together as cancer chemotherapy medication.

Valsartan has significant blood pressure lowering effect via modulating renin-angiotensin system although its mechanism of action in herceptin-induced myocardial injury is largely unknown. Cardiac TnI has been used to indicate cardiomyocyte injury and hsCRP to indicate presence of inflammation. We therefore evaluated the effect of valsartan in herceptin-induced cardiotoxicity in breast cancer patients using cTnI, hsCRP, and EF\% as markers for cardiotoxicity.
The cumulative dose of Adriamycin has been shown to cause chronic cardiotoxicity which shows up within a year after administration, the latter is known to contribute to dilated cardiomyopathy-like congestive heart failure [4].

In this study, we aimed to protect cancer patients against cardiotoxicity and eventual cardiac dysfunction by determining the potential utility of cardioprotective valsartan and to use biomarkers to assess the protective effect of valsartan.

Although the use of Adriamycin and herceptin has been associated with important gains in survival in HER2-positive cancer patients, however, their use has been associated with a significant risk of cardiotoxicity and morbidity.

*Corresponding author: Zuhair Allebban, Middle Euphrates Unit fo Cancer Research, College of Medicine/University of Kufa, Najaf, Iraq, Tel: +009647804272223; E-mail: zuhair.allabban@uokufa.edu.iq

Received March 15, 2018; Accepted May 02, 2018; Published May 04, 2018

Citation: Al-Hamadi NA, Al-Sabri I, Aldujele AB, Al-Fatlawi J, Allebban Z (2018) Valsartan Attenuates Cardiotoxicity in Breast Cancer Patients After Chemotherapy. J Cancer Sci Ther 10: 105-109. doi: 10.4172/1948-5956.1000526

Copyright: @ 2018 Al-Hamadi NA, et al. This is an open-access article distributed under the terms of the Creative Commons Attribution License, which permits unrestricted use, distribution, and reproduction in any medium, provided the original author and source are credited. 
Previous studies showed that cardiac dysfunction after treatment with a combination of Adriamycin and herceptin resulted in 18\% incidence of cardiac dysfunction, with $2 \%-4 \%$ incidence of severe symptomatic heart failure [5-7]. Analysis of data from the Cancer Research Network revealed that treatment with a combination of anthracyclines and herceptin was associated with a $>7$-fold increase in heart failure and cardiomyopathy risk [8].

Herceptin inhibits the effects of overexpression of HER2. If the breast cancer does not overexpress HER2, herceptin will have no beneficial effect and may cause harm. Doctors use laboratory tests to determine whether HER-2 is overexpressed.

Heart disease patients or patients that are also receiving other cancer medications, such as Adriamycin, can suffer heart failure. Identification of predisposed cancer patients and those that are at high risk for cardiac complications is utmost important. Such patients require early identification of subclinical cardiac dysfunction and the necessary cardioprotective strategies to ensure the continuation of their cancer therapy and to reduce their risk of heart failure. More efficient methods are needed to identify patients at higher risk of cardiotoxicity. The traditional method of using left ventricular ejection fraction (LVEF) for screening of patients before treatment and during therapy [9] has not shown sensitivity in detecting early subclinical changes or later decline in cardiac function $[10,11]$.

Detection of preclinical cardiotoxicity requires newer more sensitive methods in high risk patients. Assessment of adverse outcomes in such patients can be more successful if multiple biomarkers are used, such strategy has been successfully employed in other cardiovascular diseases [8-10]. Our aim was to determine if the serum level of any one of the biomarkers and the early changes in their levels, or a combination of biomarkers levels along with EF\% could predict subsequent cardiotoxicity in patients treated with adriamycin and herceptin.

\section{Materials and Methods}

\section{Study population}

A total of 60 untreated breast cancer patients (ages $24-70$ yrs, mean 56) were enrolled in this study. Group 1 had 30 patients, they received ACH plus valsartan, group 2 had also 30 patients and received only $\mathrm{ACH}$.

Patients were scheduled to receive chemotherapy at the Middle Euphrates Cancer Therapy Center in Najaf, Iraq from February 2016 to February 2017.

The study population consisted of patients with HER2-positive breast cancer recruited at the Cancer Therapy Center in Najaf, Iraq and were randomly assigned to groups. Patients were included in the study if they were untreated patients with HER2-positive early-stage breast cancer that was either spread to the lymph node or not spread to the lymph nodes. All were scheduled to undergo adjuvant therapy with an adriamycin and cyclophosphamide-containing regimen followed by taxotere and herceptin (Genentech, San Francisco, California, USA) as outlined in Figure 1. At 3 months, valsartan $(8 \mathrm{mg} / \mathrm{kg} /$ day $)$ was administered concurrently with herceptin ( $6 \mathrm{mg} / \mathrm{kg}$ per two days) for 12 weeks, herceptin was then continued to be administered every 3 weeks to complete 1 year of therapy.

According to the study protocol outlined in Figure 1, both group 1 and 2 received 4 cycles of Adriamycin $\left(60 \mathrm{mg} / \mathrm{m}^{2}\right)$ and cyclophosphamide $\left(600 \mathrm{mg} / \mathrm{m}^{2}\right)$ followed by 21 days of taxotere $\left(60 \mathrm{mg} / \mathrm{m}^{2}\right)$ and herceptin (loading dose $8 \mathrm{mg} / \mathrm{kg} /$ day, and $6 \mathrm{mg} / \mathrm{kg} /$ day for 21 days). Group 1

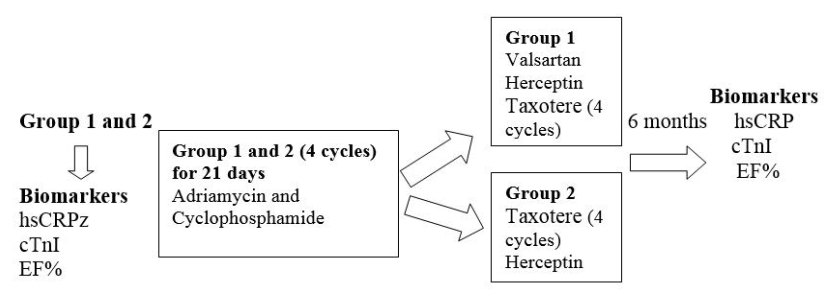

Figure 1: Study protocol for participants before and after chemotherapy with/ without valsartan. Data were obtained from participants before chemotherapy and at 6 months after Adriamycin, cyclophosphamide, taxotere and herceptin therapy using echocardiograms and serum hsCRP, serum cTnl and EF\%. These parameters were measured at baseline, and at 6 months after valsartan and chemotherapy drug administration.

received valsartan $(8 \mathrm{mg} / \mathrm{kg} /$ day $)$ and herceptin $(6 \mathrm{mg} / \mathrm{kg} /$ day $)$, group 2 received 4 cycles taxotere $\left(75 \mathrm{mg} / \mathrm{m}^{2}\right)$ and herceptin $(6 \mathrm{mg} / \mathrm{kg} /$ day $)$. Six months later all patients in group 1 and 2 were tested for serum hsCRP, serum cTnI, and for $\mathrm{EF} \%$.

\section{Exclusion criteria}

Pregnant and nursing mothers were excluded from the study. Other exclusion criteria were used on patients with the following conditions: a contraindication for angiotensin-II antagonists, uncontrolled diabetes mellitus, cerebral vascular accidents within the past 3 months, hemodynamically significant valve disease, severe psychopathy, cirrhosis, other cardiac diseases (coronary artery disease, atrial arrhythmias, arrhythmia needing medication, dilated cardiomyopathy), noncompliance and treatment with any of the following drugs within the past 3 months: ACE inhibitors, vitamin E, angiotensin-II antagonists, probucol, calcium antagonists, $\beta$-blockers, or steroid pulse therapy. In addition to the above exclusions, patients with an LVEF $<50 \%$ before chemotherapy or those who were unwilling or unable to provide informed consent were excluded too.

Participants were studied before chemotherapy and at 6 months after ACH therapy using echocardiograms for measuring LVEF and blood samples to measure serum cTnI and hsCRP. Blood samples were obtained before treatment and at 6 months as outlined in Figure 1 and were kept at $-80^{\circ} \mathrm{C}$ until tested. A reduction in LVEF of $\geq 5 \%$ to $<55 \%$ with symptoms of heart failure or an asymptomatic reduction of LVEF of $\geq 10 \%$ to $<55 \%$ were used as endpoint of cardiotoxicity as is defined by the Cardiac Review and Evaluation Committee [1]. All participants provided written or verbal informed consent, and the protocol was approved by the institutional review board of the college of medicine/ Kufa university, Najaf, Iraq.

\section{Echocardiography}

Measurement of LVEF is considered to be an essential measurement in heart failure (HF). Ejection fraction is used to assess LV function in more situations than just treatment of cardiovascular diseases. Digital echocardiograms (Seimens Medical solution, USA, Acuson CV70 GM-5670ADOE Model no. 07848521) was analyzed on the EchoPAC workstation (GE Healthcare). Left ventricular end-diastolic and -systolic volumes were obtained using Simpson's method of discs in the apical 4- and 2-chamber views as recommended by the American Society of Echocardiography [12]. Calculation of ejection fraction from left ventricular volumes was obtained using this formula: (end-diastolic volume - end-systolic volume)/end-diastolic volume $\times 100 \%$. Tracings were made by a single observer who was blinded to all other clinical or biomarker data. 


\section{Laboratory analyses}

Blood samples were collected from patients in EDTA and lithium heparin-containing tubes, plasma was obtained after spinning the tubes at $3000 \mathrm{rpm}$ for 15 minutes, aliquoted, and stored at $-80^{\circ} \mathrm{C}$ until the time of testing. Plasma samples were thawed only once before use. Measurement of cTnI was performed on the Dimension Vista 500 Intelligent Laboratory System (Siemens Healthcare Diagnostics, Deerfield, Illinois, USA), hsCRP was measured with a standard Architect immunoassay as detailed by the manufacturer (Abbott Laboratories, Abbott Park, Illinois). Coefficient of variation (CV) for all assays were at $<10 \%$. Briefly, cTnI had a $10 \% \mathrm{CV}$ at $3 \mathrm{ng} / \mathrm{L}$, and hsCRP at $\leq 6 \%$. Cardiac TnI was measured following the manufacturer protocol.

Estimation of protein expression using Enzyme Linked Immunosorbent Assay (ELISA) technique was used for estimation of the level of CA 15-3 (CODA Automated EIA Analyzer, by using MP Biomedicals (USA) Kit following the manufacturer protocol. Immunohistochemistry was done for estrogen receptor (ER), progesterone receptor (PR), and HER2 expression studies following the manufacturer protocol.

\section{Statistical methods}

Statistical analysis was done using SPSS (Statistical Package for Social Sciences) version 20 , in which we use number with percentages and mean with standard deviation as descriptive statistics. For analytic statistics we use chi-square test for categorical variables and independent sample $\mathrm{t}$-test for continuous variables. $\mathrm{P}$ value at $\leq 0.05$ was regarded significant.

\section{Results}

All of the 60 patients included in this study were HER2-positive breast cancer patients. The patients were divided into two groups with 30 in each group. The mean age of patients in group 1 was $52.7 \pm 11.83$ years which is significantly different from the mean age of group 2 which was $45.93 \pm 12.08$ years $(\mathrm{p}=0.023)$ as shown in Table 1 . All the study participants were positive for invasive ductal carcinoma and only one patient was positive for medullary ductal carcinoma, and there was no significant difference between group 1 and 2 with regard to the type of tumor and also to the presence and absence of both PR and ER as presented in Tables 1 and 2.

There was a significant increase in hsCRP and EF\% in group 2 compared to group $1(\mathrm{p} \leq 0.001)$, and there was significant decrease in group 2 compared to group 1 as shown in Table 3 . Statistical analysis showed no significant association between baseline serum levels of biomarkers and cardiotoxicity except for cTnI.

\section{Discussion}

Due to the cardiotoxicity of adriamycin and herceptin used for treatment of breast cancer patients, cardiac dysfunction has been diagnosed in 1 of 4 women treated with these anti-cancer drugs [1318]. Breast cancer is the most common female malignancy comprising

\begin{tabular}{|c|c|c|c|c|c|}
\hline \multirow{2}{*}{\multicolumn{2}{|c|}{ Variables }} & \multicolumn{2}{|c|}{ Groups } & \multirow{3}{*}{$\begin{array}{c}\text { Total } \\
60\end{array}$} & \multirow{2}{*}{ P-value } \\
\hline & & Group $1(n=30)$ & Group $2(n=30)$ & & \\
\hline \multirow{4}{*}{ Type } & \multirow{2}{*}{ Invasive Ductal Carcinoma } & 30 & 30 & & \multirow{4}{*}{0.313} \\
\hline & & $49.2 \%$ & $50.8 \%$ & $100.0 \%$ & \\
\hline & \multirow{2}{*}{ Medullary Ductal Carcinoma } & 1 & 0 & 1 & \\
\hline & & $100.0 \%$ & $0.0 \%$ & $100.0 \%$ & \\
\hline \multirow{4}{*}{ ER } & \multirow{2}{*}{ Negtive } & 13 & 19 & 32 & \multirow{4}{*}{0.121} \\
\hline & & $40.6 \%$ & $59.4 \%$ & $100.0 \%$ & \\
\hline & \multirow{2}{*}{ Postive } & 17 & 11 & 28 & \\
\hline & & $60.7 \%$ & $39.3 \%$ & $100.0 \%$ & \\
\hline \multirow{4}{*}{ PR } & \multirow{2}{*}{ Negtive } & 10 & 17 & 27 & \multirow{4}{*}{0.069} \\
\hline & & $37.0 \%$ & $63.0 \%$ & $100.0 \%$ & \\
\hline & \multirow{2}{*}{ Postive } & 20 & 13 & 33 & \\
\hline & & $60.6 \%$ & $39.4 \%$ & $100.0 \%$ & \\
\hline Age (years) & -- & $52.7 \pm 11.83$ & $45.93 \pm 12.08$ & 60 & 0.023 \\
\hline CA15-3 & -- & $48.02 \pm 44.66$ & $45.83 \pm 33.05$ & 60 & NS \\
\hline
\end{tabular}

Table 1: Distribution of estrogen and progesterone surface receptors among study participants.

\begin{tabular}{|c|c|c|c|c|c|c|}
\hline \multirow{2}{*}{ Parameters } & \multicolumn{3}{|c|}{ Group 1 (M+SD) } & \multicolumn{3}{|c|}{ Group 2 (M+SD) } \\
\hline & Before treatment & After treatment & $P$ - value & Before treatment & After treatment & P-value \\
\hline hsC-reactive protein & $27.1 \pm 4.9$ & $5.6 \pm 2.0$ & $\leq 0.001$ & $39.9 \pm 7.4$ & $9,9 \pm 2.8$ & $\leq 0.001$ \\
\hline cTroponin I & $0.15 \pm 0.09$ & $6.1 \pm 4.7$ & $\leq 0.001$ & $1.5 \pm 0.86$ & $31.0 \pm 14.5$ & $\leq 0.001$ \\
\hline Ejection fraction \% & $62.0 \pm 3.4$ & $61.0 \pm 3.2$ & $\leq 0.001$ & 63.263 .2 & $59.0 \pm 59.0$ & $\leq 0.001$ \\
\hline
\end{tabular}

Table 2: Biomarkers of patients treated with six sessions of valsartan and anti-cancer drugs (Group 1), and patients treated with six sessions of only anti-cancer drugs (Group 2).

\begin{tabular}{|c|c|c|}
\hline \multirow{2}{*}{ Variables } & Group 1 (n=30) & Group 2 (n=30) \\
\cline { 2 - 3 } & Mean difference \pm SD & Mean difference \pm SD \\
\hline hsC-reactive protein & $-21.53 \pm 5.78$ & $-30.06 \pm 7.55$ \\
\hline cTroponin I & $5.95 \pm 4.70$ & $29.53 \pm 14.54$ \\
\hline Ejection fraction $\%$ & $-1 \pm 0.69$ & $-4.23 \pm 0.97$ \\
\hline
\end{tabular}

Table 3: Comparison of mean difference in all parameters under study before and after 6 treatment sessions in group 1 and 2 . 
the second leading cause of cancer-related deaths in Iraqi women. HER2 positive breast cancer patients can benefit from treatment with herceptin, but its cardiotoxicity and its long-term effects are of concern in such patients. In most cases, reduction of cardiac function after treatment with herceptin is reversible, some patients, especially those with cardiac risk factors, may rarely experience chronic heart failure or prolonged LVEF reduction as demonstrated in our study. In this study, we managed to reduce adriamycin/herceptin induced cardiotoxicity by the prophylactic administration of valsartan as characterized by improvement of EF\% and by lowering serum level of cTnI and hsCRP.

Ejection fraction is used to assess function in more situations than just treatment of cardiovascular diseases. The administration of several potent chemotherapeutic agents is largely guided by the potential occurrence of cardiotoxicity, mainly defined by decrementing LVEF values [7]. In a previous study, $14 \%$ of patients were withheld further treatment with potentially life-saving therapies such as herceptin based on asymptomatic reduction in LVEF alone [8]. Our study demonstrated both symptomatic and asymptomatic cardiotoxicity as previously reported $[7,8]$, but valsartan demonstrated its efficacy as a prophylactic drug for cardiotoxicity.

Assessment of cardiotoxicity, both before and during therapy is essential in high risk patients to reduce morbidity and mortality in such patients, and to increase the chance of a successful and safe treatment. The risk of cardiotoxicity was not significantly associated with baseline levels of any biomarker, except for cTnI as has been previously established [13].

In this study, we found that post-treatment cardiotoxicity can be predicted by using multiple markers, including cTnI, hsCRP, and EF\%. We also showed that early changes in cTnI or hsCRP serum levels or $\mathrm{EF} \%$ were found to be associated with subsequent cardiotoxicity.

The cardiotoxicity associated with herceptin was first reported in metastatic breast cancer cases, with $22 \%$ of patients receiving herceptin experiencing symptomatic or asymptomatic cardiac dysfunction [14]. While the drug has significant therapeutic effect, the cardiotoxicity of herceptin, ranging from asymptomatic left ventricular dysfunction to congestive heart failure, has emerged as a critical concern in clinical practice [15-17]. Herceptin-related cardiotoxicity has been considered dose-independent and highly reversible even when the drug is continued, in contrast with anthracycline regimens [8].

Biomarkers have been shown to be helpful for the detection of subclinical cardiotoxicity in various malignancies undergoing treatment with $\mathrm{ACH}$. When treated with high dose of anthracycline, 703 patients with various malignancies, showed the value of biomarkers as predictive tool for cardiotoxicity [16]. This is similar to the results of our study which showed a significant increase in serum cTnI value during cardiotoxicity.

In a similar study by the same group of investigators, they were able to show that elevation in the level of cTnI correlated with cardiotoxicity. Their subjects were breast cancer patients that were treated with herceptin, with $78 \%$ of these patients were previously exposed to anthracycline [17].

However, the rise in the level of cTnI, was not specific in another study in which the rising biomarker showed no association with the change in LVEF, the patients in that study, had this biomarker elevated during their sequential therapy with doxorubicin, trastuzumab, and lapatinib [18], these results are similar to the results of our study. Our data also demonstrated an increase of CA15-3 in all study participants.
It has been reported that persistent elevation in CA15-3 is considered a reliable prognostic marker in HER-2 positive breast cancer as they are directly related to advanced stages and recurrence, our results confirmed that association.

Our investigation suggested that monitoring cTnI level could be an effective tool to detect anysubsequent cardiac dysfunction in cancer patients being treated with chemotherapy. After initial adriamycin and cyclophosphamide chemotherapy, we used baseline level of cTnI and its subsequent measurement as a tool to evaluate the risk of cardiotoxicity. We found an association of cTnI levels and subsequent cardiotoxicity.

We suggest that future clinical breast cancer research should be adequately powered, with larger sample size and longer follow-up. Also the patients need to be evaluated every 3 months during their cancer therapy to assess the true efficacy, toxicity and later the survival rate. This could provide a comprehensive understanding of cardiotoxicity associated with long-term herceptin administration and the role of valsartan as a prophylactic drug against cardiotoxicity. Additionally, other biomarkers of cardiotoxicity demonstrated benefits in other studies, need to be included in future studies such as serum $\mathrm{N}$-terminal pro-B-type natriuretic peptide (NT-proBNP) and myeloperoxidase.

\section{Conclusion}

Our study confirms the value of the prophylactic use of valsartan in reducing the cardiotoxic effect in breast cancer patients treated with chemotherapeutic agents as characterized by improvement in EF\% level and by reducing cTnI and hsCRP serum levels. We also conclude that $\mathrm{cTnI}$ could serve as a potential tool in assessing cardiotoxicity and subsequent cardiac dysfunction in patients undergoing sequential chemotherapy with $\mathrm{ACH}$.

\section{Conflict of Interest Statement}

The authors declare that there is no conflict of interest.

\section{Author's Contributions}

NAA, conceived and designed the experiments. IA, designed the experiment and contributed clinically. $\mathrm{AAB}$, contributed to the design of the experiments and laboratory testing. SJA, performed the statistical analysis and reviewed the manuscript. ZA, contributed to the design, writing, and critical review of the manuscript. All authors reviewed and approved the final manuscript.

\section{References}

1. Pangilinan JM (2013) Trastuzumab (Herceptin) use during pregnancy: Is Trastuzumab Safe During Pregnancy? Medscape.

2. AHSP (1942) The American Society of Health-System Pharmacists

3. Guarneri V, Lenihan DJ, Valero V, Durand JB, Broglio K, et al. (2006) Longterm cardiac tolerability of trastuzumab in metastatic breast cancer: The M.D. Anderson Cancer Center experience. J Clin Oncol 24: 4107-4115.

4. Jensen BV, Skovsgaard T, Nielsen SL (2002) Functional monitoring of anthracycline cardiotoxicity: A prospective, blinded, long-term observational study of outcome in 120 patients. Ann Oncol 13: 699-709.

5. Slamon D, Eiermann W, Robert N, Pienkowski T, Martin M, et al. (2002) Cardiac dysfunction in the trastuzumab clinical trials experience. J Clin Oncol 20: 1215-1221.

6. Suter TM, Procter M, Van Veldhuisen DJ, Muscholl M, Bergh J, et al. (2007) Trastuzumab-associated cardiac adverse effects in the Herceptin adjuvant trial. J Clin Oncol 25: 3859-3865.

7. Slamon D, Eiermann W, Robert N, Pienkowski T, Martin M, et al. (2011) Adjuvant trastuzumab in HER2-positive breast cancer. N Engl J Med 365: 1273-1283. 
Citation: Al-Hamadi NA, Al-Sabri I, Aldujele AB, Al-Fatlawi J, Allebban Z (2018) Valsartan Attenuates Cardiotoxicity in Breast Cancer Patients After Chemotherapy. J Cancer Sci Ther 10: 105-109. doi: 10.4172/1948-5956.1000526

8. Bowles EJ, Wellman R, Feigelson HS, Onitilo AA, Freedman AN, et al. (2012) Risk of heart failure in breast cancer patients after anthracycline and trastuzumab treatment: A retrospective cohort study. J Natl Cancer Inst 104 1293-1305.

9. Altena R, Perik PJ, Van Veldhuisen DJ, De Vries EG, Gietema JA (2009) Cardiovascular toxicity caused by cancer treatment: strategies for early detection. Lancet Oncol 10: 391-399.

10. Swain SM, Whaley FS, Ewer MS (2003) Congestive heart failure in patients treated with doxorubicin: a retrospective analysis of three trials. Cancer 97: 2869-2879.

11. Lang RM, Bierig M, Devereux RB, Flachskampf FA, Foster E, et al. (2005) Recommendations for chamber quantification: a report from the American Society of Echocardiography's guidelines and standards committee and the chamber quantification writing group. J Am Soc Echocardiogr 18: 1440-1463.

12. Sawaya H, Sebag IA, Plana JC, Januzzi JL, Ky B, et al. (2012) Assessment of echocardiography and biomarkers for the extended prediction of cardiotoxicity in patients treated with anthracyclines, taxanes and trastuzumab. Circ Cardiovasc Imaging 5: 596-603.

13. Slamon DJ, Leyland-Jones B, Shak S, Fuchs H, Paton V, et al. (2001) Use of chemotherapy plus a monoclonal antibody against HER2 for metastatic breast cancer that overexpresses HER2. N Engl J Med 344: 783-792.
14. Piccart-Gebhart MJ, Procter $M$, Leyland-Jones B, Goldhirsch A Untch $M$ et al. (2005) Trastuzumab after adjuvant chemotherapy in HER2-positive breast cancer. N Engl J Med 353: 1659-1672.

15. Romond EH, Jeong JH, Rastogi P, Swain SM, Geyer CE Jr, et al. (2012) Seven-year follow-up assessment of cardiac function in NSABP B-31, A randomized trial comparing doxorubicin and cyclophosphamide followed by paclitaxel (ACP)with ACP plus trastuzumab as adjuvant therapy for patients with node-positive, human epidermal growth factor receptor 2-positive breast cancer. J Clin Oncol 30: 3792-3799.

16. Beda M, Basso U, Ghiotto C, Monfardini S (2007) When should trastuzumab be stopped after achieving complete response in HER2-positive metastatic breast cancer patients? Tumori 93: 491-492.

17. Putt M, Hahn VS, Januzzi JL, Sawaya H, Sebag IA, et al. (2015) Longitudina changes in multiple biomarkers are associated with cardiotoxicity in breast cancer patients treated with doxorubicin, taxanes, and trastuzumab. Clin Chem 61: 1164-1172.

18. Hashim ZM (2014) The significance of CA15-3 in breast cancer patients and its relationship to HER-2 receptor status. Int $\mathrm{J}$ Immunopathol Pharmacol 27: $45-51$ 